\title{
The Panel Test as the Metrology of Extra Virgin Olive Oil Quality Evaluation and Its Dissemination
}

\author{
Maurizio Caciotta ${ }^{1}$, Sabino Giarnetti ${ }^{1}$, Fabio Leccese ${ }^{1}$, Barbara Orioni ${ }^{1}$, Marco Oreggia ${ }^{2}$ and Salvatore Rametta ${ }^{1}$ \\ 1. Science Department, University of “Roma Tre”, Rome 00146, Italy \\ 2. E.V.O. srl, Rome 00134, Italy
}

\begin{abstract}
Nowadays, the check of the organoleptic characteristics for the evaluation of extra virgin olive oil (EVOO) quality is regulated by the European Union (EU) authorities, which indicate the use of the panel test (PT). It is composed by a team of specialists that give a numerical value to many characteristics about flavours, synthesising a sensory analysis. Each expert answers questions about the aroma by assigning the adequate scores to each oil. The evaluation becomes objective by applying the statistical analysis of all the scores given by the participants: This is the definition of "measure" of Russell. The PT can be considered a true standard "metrological system" (considering the number of questions in the questionnaire), while the perceptions of the testers are the solicitations of it. To allow access to an expensive evaluation process by small companies, this work proposes to "disseminate" the properties of the metrology represented by PT. The results of the PT are arranged in an unsupervised artificial neural network (ANN), the Kohonen map, which represents the synthesis of self-organised output that has only the goal, in this paper, to make readable PT results. The dissemination process is obtained by the gas chromatographic (GC) analysis of each oil sample and through the identification of peaks corresponding to the perceptions. These signals are used for the training of the supervised Multi Layer Perceptron (MLP) ANN, with the back propagation algorithm, whose outputs are represented by the results of the PT. This procedure is exact a "metrological dissemination of a standard" and also the aim of the work: to classify EVOO without always resorting to PT.
\end{abstract}

Key words: EVOO, quality evaluation, gas chromatography, ANN.

\section{Introduction}

The quality of goods is lexically defined as the "level of excellence". The International Organisation for Standardisation (ISO), in 9001 norm, defines quality as "whatever the customer perceives good quality to be" [1]. The large increase in demand for high quality extra virgin olive oil (EVOO) is due to its peculiar characteristics that play an important role in human nutrition. The concept of its quality is not unique and the discussion within the scientific and technical community is extremely animated: EVOO quality may be defined from productive, commercial, nutritional and organoleptic points of view [2,3].

Olive oil is the first food product which the assessment of the organoleptic quality used the panel

Corresponding author: Barbara Orioni, Ph.D., research field: analytical chemistry. E-mail: b.orioni@yahoo.it. test (PT) introduced at the level of European Union (EU) legislation as a criterion for the classification. The sensory evaluation of olive oil is now carried out according to the method described in the Reg. EU 1348/2013 annex XII (revision of Reg. EEC 2568/91) [4]. Usually, the PT is used to define the quality of the virgin olive oil rather than the intrinsic ability to analyse the relative organoleptic characteristics [5-8]. This work presents a "metrological system", specific for EVOO, based on the evaluation of the panelists, the only custodian of the quality definition, in accordance to the norm. The PT is the standard of the organoleptic qualities and we propose the implementation of a metrology dissemination system, typical of all the scientific processes.

The PT is a sensory analysis carried out by a team of experts (from 8 to 12 panelists) which, through the tasting, give a numerical subjective evaluation (from 0 
to 5) to many questions about flavours, in a questionnaire, for several EVOO samples. This system uses human sense as a measurement instrument, because they are naturally able to interpret the complex chemical structure of EVOO and translating it in stimulus which involves the human senses. The subjectivity is removed by statistical methods applied to all the panelists' scores and the obtained values, for each question, represent the measure of flavours component, according to Russell [9].

The ensemble of the scores, reached by each components, defines the organoleptic quality. These scores suggest to use an unsupervised artificial neural network (ANN), which identifies the best two-dimensional representation of the samples that has stimulated the PT.

By utilising gas chromatographic (GC) analysis, with headspace sampling, this system will be the one that is the closest to the procedure for judging the organoleptic quality compared with the panelists.

The GC is able to separate and identify volatile compounds of the olive oil, which are responsible for the flavour and taste perception [3, 1-13], with the advantages of greater sensitivity. In fact, the volatile components are concentrated on the vapour phase where substances decomposition, solvents or other chemicals are not present, so the sample is not contaminated and the analysis is not affected.

The purpose of the proposed system is to "disseminate metrologically", the organoleptic classification of EVOO keeping the PT as metrological reference utilising a supervised $\mathrm{ANN}$, in order to combine GC and PT results.

During the training step, the supervised ANN stores the analytical capacity of the PT in its parameters [14, 15]. An EVOO, out of the set of samples utilised by PT to define quality and submitted to GC, whose results are the input of the supervised $\mathrm{ANN}$, can be correctly classified in the unsupervised two-dimensional ANN like as if it had been submitted directly to the PT, without this actually occurring.

\section{Materials and Methods}

\subsection{Materials}

The proposed method was applied to nine EVOO, eight of which are of high quality monocultivar and industrial ones. Monocultivar EVOO are from different Italian regions, and the olive varieties, with their reference symbols are: AscolanaTenera (a, A), Casaliva (b, B), TondaIblea (c, C), Ottobratica (d, D), OlivastraSeggianese (e, E), Carolea (f, F), Nocellara del Belice (g, G), Frantoio (i, I) [16]. Industrial sample is identified by $\mathrm{h}$ and $\mathrm{H}$ letters.

\subsection{Methods}

The standard establishes that olive oil must be tasted according to the rigid standards and regulations of the PT that is carried out by a group of at least eight professional tasters with a chief. The components of the PT are required to provide a numerical rating, ranging between 0 and 5, for a series of flavours, which are listed in a sheet. The table used in this work contains more questions than EU model: This choice is dictated by a desire to enhance the possible correlations between the flavours. Positive and negative attributes and other perceptions are listed in EU regulation [4].

Aromatic analysis is carried out by smelling the sample, taking slow deep breaths to evaluate the oil, with a pause between each breath. Then, the taster shall then evaluate the gustatory and retronasal sensations by taking a small sip of oil. It is very important to distribute the oil throughout the whole of the mouth cavity.

The members of the PT follow a training of six months before being a reliable component of the panel itself. They have to pass some exams as pre-selections and exams during the lessons of training. In order to deepen the metrological value of the PT, reserve the regulation, a more extended questionnaire than that provided by the EU sheet was prepared. In the present 
work, static head space (HS) sampling was applied [17]. HS-GC analyses have been performed by placing a $1.8 \mathrm{~g}$ sample of EVOO into a $10 \mathrm{~mL}$ headspace vial. Then the vial has been hermetically sealed with a PTFE (Polytetrafluoroethylene, best known as Teflon $^{\circledR}$ ) septum and pre-incubated at $40{ }^{\circ} \mathrm{C}$ for $1 \mathrm{~h}$; this simulates the oropharyngeal cavity, where the flavours develop. The vapour in equilibrium with sample has been extracted by a Hamilton syringe and immediately inserted into the injection port of the GC system at $250{ }^{\circ} \mathrm{C}$ and in splitless mode (pressure 25 psi). Analyses were performed using an Agilent 7820A Gas Chromatograph (Agilent Technologies, Santa Clara USA) using helium carrier gas at a flow rate of $6.5 \mathrm{~mL} / \mathrm{min}$ and a Flame Inoization Detector (FID) detector maintained at $300{ }^{\circ} \mathrm{C}$. The volatile fraction has been transferred into a non-polar capillary column (5\% phenyl, $95 \%$ dimethylpolysiloxane, $30 \mathrm{~m}$, $0.32 \mathrm{~mm}$ i.d., $0.25 \mu \mathrm{m}$ film thickness). The oven temperature is held at $75{ }^{\circ} \mathrm{C}$ for $30 \mathrm{~s}$ and programmed to rise at $20{ }^{\circ} \mathrm{C} / \mathrm{min}$ to a final temperature of $200{ }^{\circ} \mathrm{C}$, at which it is held for $120 \mathrm{~s}$. On a quality level, many of the signals have been assigned to organic volatile molecules by comparing our gas chromatograms to Agilent database and, consequently, correlated to characteristic flavour [18] in accordance to other authors which used a similar column [19].

In unsupervised training, the networks learn to form their own classifications of the training data without external help. The unsupervised network [20] is designed to perform a non-linear mapping of a high-dimensionality data space, transforming it in a low-dimensional space, usually 2D. This representation is the Kohonen map. The visualisation of the output data is performed from the distance/proximity of neurons in an output grid. The output neurons are only connected to its nearest neighbours. The proximity represents a similar pattern represented by an output neuron. The system is based on competitive learning, in which the output neurons compete amongst themselves to be activated, with the result that only one is activated at any one time. This activated neuron is called the winning neuron. The result is that the neurons are forced to organise themselves. For obvious reasons, such a network is called a self organising map (SOM). The structure is shown in Fig. 1.

This type of ANN maps sets input data onto a set of appropriate outputs, and it is a supervised network because it requires a desired output in order to learn. A multi layer perceptron (MLP) consists of multiple layers of nodes in a directed graph, with each layer fully connected to the next one. The MLP uses an algorithm called back-propagation (BP), which uses the data in order to modify the weights and other parameters to minimise the prediction "learning error". This error decreases with each iteration and the neural model gets closer and closer to producing the desired output. This process is known as "training" [21]. The supervised learning, given a set of example pairs $(X$, $Y$ ), has the purpose of finding a function $f: X \rightarrow Y$ in the allowed class of functions that matches the examples. It has three-layered system: an input layer, intermediate called hidden layer and an output one, as shown in Fig. 2.

\section{Results and Discussion}

The main parameters that identify the best oils are fruity, green, bitter, spicy and sweet qualities. Fig. 3 shows the results of PT. Each perception is represented in accordance to the average value of the assigned score.

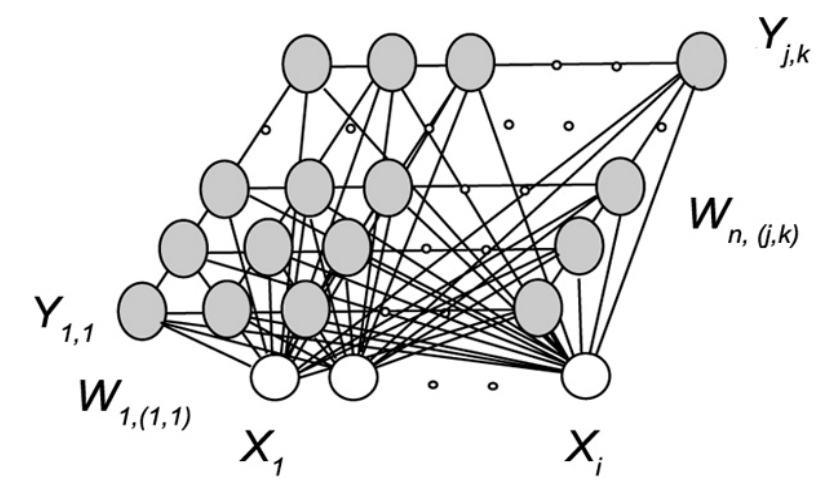

Fig. 1 Structure of unsupervised ANN. 


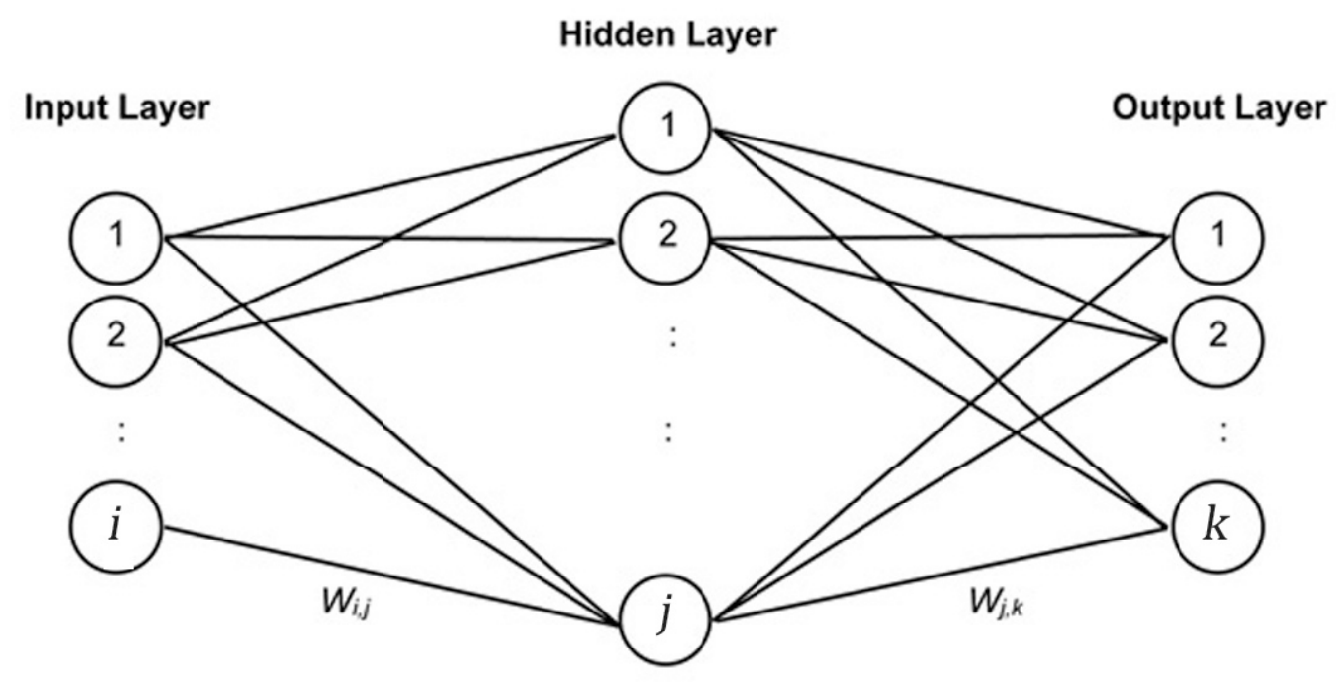

Fig. 2 Multi layer perceptron topology.

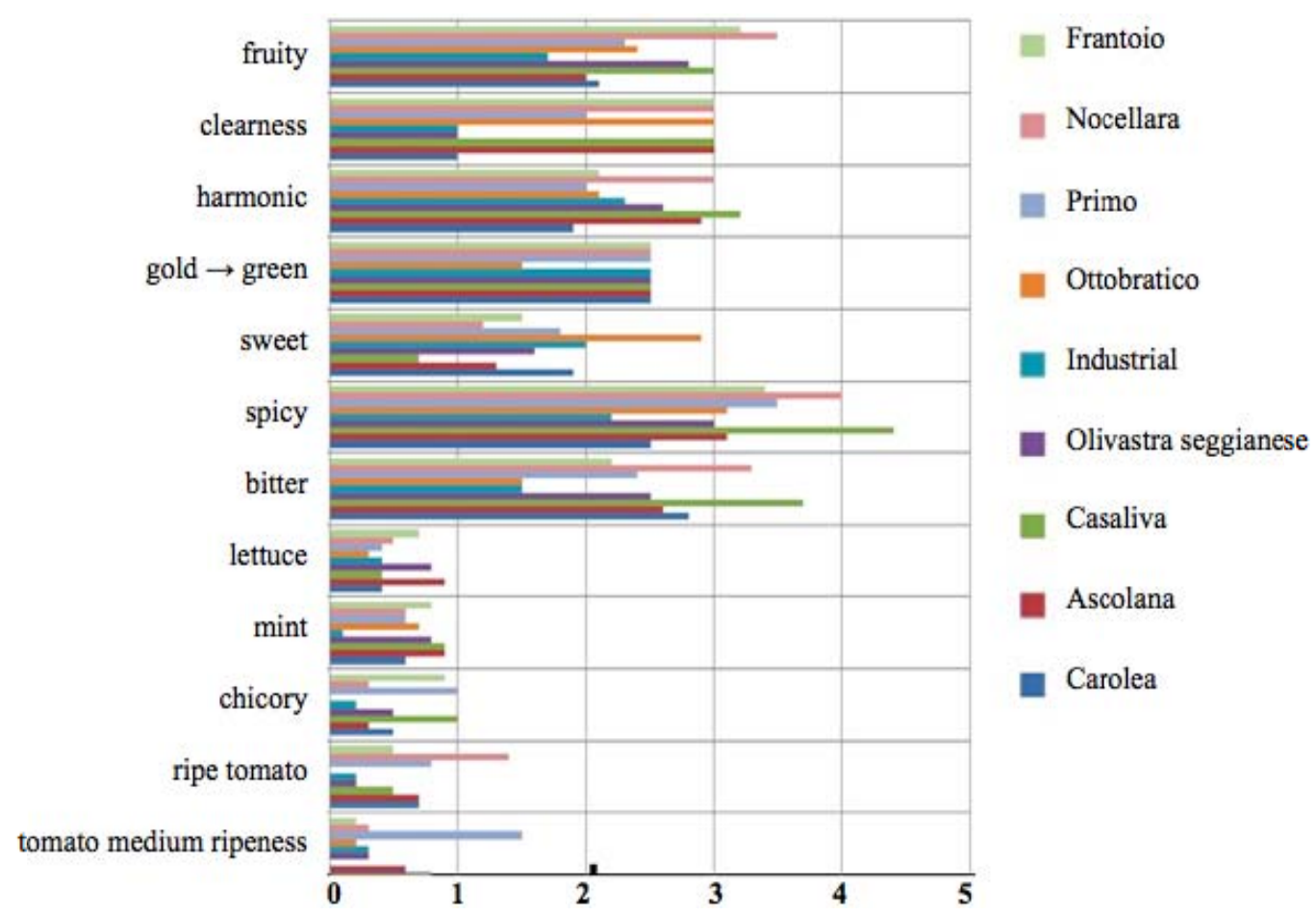

Fig. 3 Synthesis of PT evaluation.

On the left — the positive attributes; in the middle — the average values assigned by panellists; on the right— the legend of oil samples.

The volatile fraction of the oils has been analysed by detecting 37 peaks. Fig. 4 shows some results. The peaks correspond to flavours, identified by comparison with literature and Agilent technical support [17, 22].

Each peak is correlated to a molecule, the detected compounds and their relative sensory attributes are listed in Table 1.

The number of inputs of the SOM is equal to the number of perceptions on which the PT is stimulated. The output dimensions are arbitrary set in a $10 \times 10$ neurons map, and all available inputs, connected to 

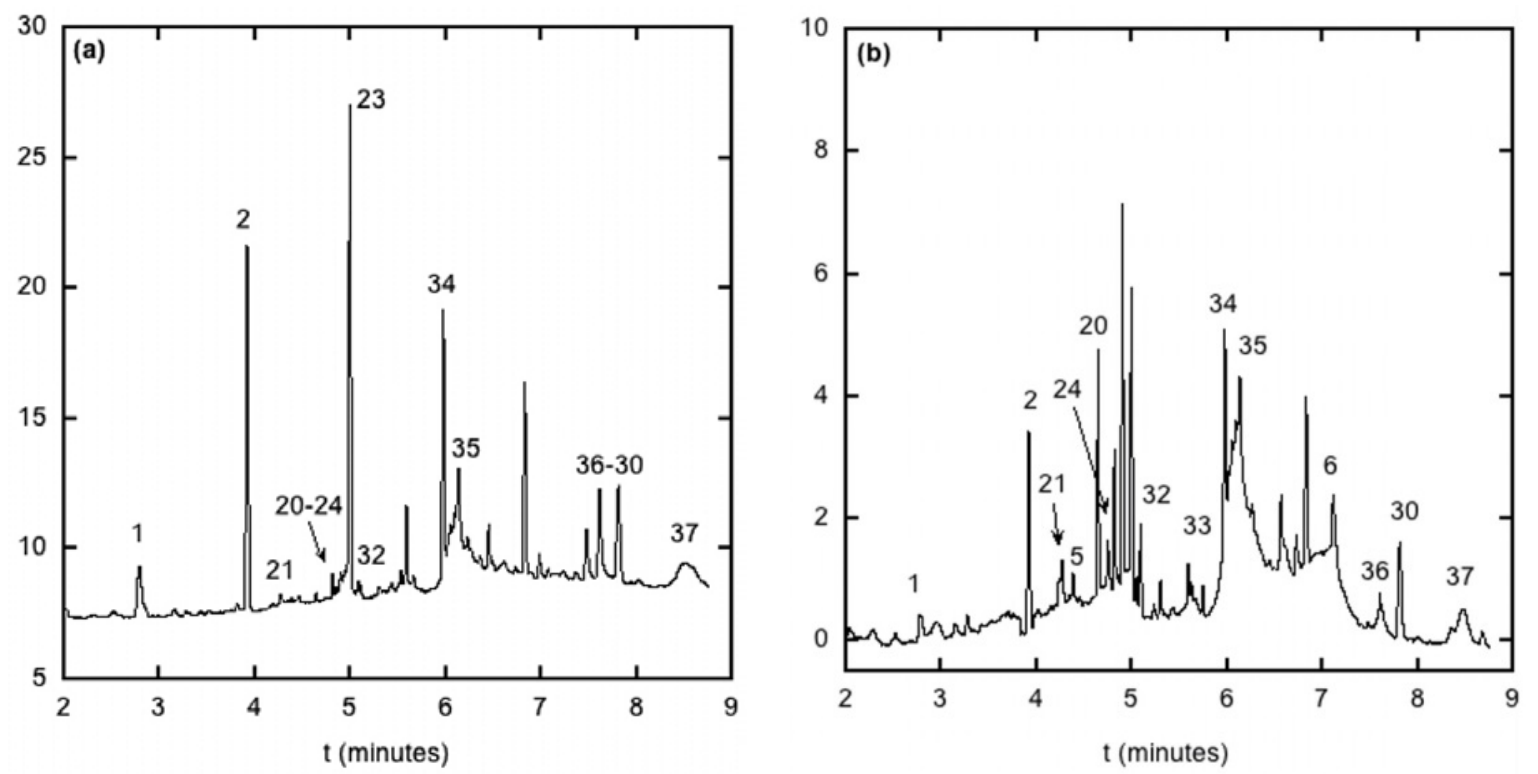

Fig. 4 (a) Sample I, Frantoio variety and (b) sample H, industrial type (arbitrary units on y-axis).

The peaks numbers correspond to flavour listed in Table 1.

oils for two cycles, one of 100 epochs with a learning rate from 0.08 to 0.02 , and the second of 1,000 cycle with a learning rate from 0.1 to 0.001 . The results are displayed in Fig. 5.

The classification in Fig. 6 shows, in map interpretation, the abscissa axis ripe-spicy develops from left to right and the y-axis ripe-sweet from the bottom to the top. The analysis can be completed by considering the sweet-fresh and spicy-fresh axis, in which oils are set according to their main characteristics. An analysis of these characteristics, highlighted by the Kohonen map, deserves in a future work a study on the meaning of the metrology system of PT.

This arrangement solves the objective problem related to the fact that the PT is a "multiple standard", seat of a large number of organoleptic characteristics which the work is addressed through the instrument of unsupervised ANN, that help a diversification in the sample that stimulate the PT and allow an internal evaluation that can be considered as its "calibration".

On the other hand, our intent is to study of the ability to "disseminate the standard" and the Kohonen map is used only as a tool for display of the response of the PT. A further work will underline the meaning of the map of Kohonen results, related to each organoleptic characteristics investigated.

To check the ability of the SOM to correct classification after the learning step, only eight of the nine oil samples were used, the sample " $i$ " was excluded. In Fig. 5, the results of the classification are shown in light-blue. The excluded sample, whose PT evaluation is known, was used to test the ability of classification of the Kohonen map and it is reported in light orange. With the aim of identifying the "objectivity" of this classification, the learning process was repeated, also taking into account the sample previously excluded. In Fig. 5, the new positioning of samples is indicated with uppercase letters on blue background.

Discrepancies will be explained in the conclusions section. It's clear that the left shift of almost all of the classifications of the samples, the sample "i" keeps its position in the lower left of the Kohonen map.

Other consideration is the marginality of industrial product $(\mathrm{h}, \mathrm{H})$, in the classification space in the Kohonen map. The vicinity with the zone in which are distributed the "quality" oils, can be explained by the 
Table 1 Volatile compounds in the EVOO samples and their corresponding aroma and flavours.

\begin{tabular}{|c|c|c|}
\hline$n$ & Volatile compound & Flavour \\
\hline 1 & O-xylene & Glue, solvent like \\
\hline 2 & $\begin{array}{l}\text { (E)-4,8-dimethyl-1,3,7-nonatri } \\
\text { ene }\end{array}$ & Flowers fragrance \\
\hline 3 & $\alpha$-copaene & Resin \\
\hline 4 & (E)-2-octene & Green grass \\
\hline 5 & Ethylbenzene & Fruity \\
\hline 6 & $(\mathrm{E}, \mathrm{E})-\alpha$-farnesene & Apple \\
\hline 7 & 6-methyl-5-hepten-2-one & Fruity \\
\hline 8 & 2-heptanone & Ripe fruits \\
\hline 9 & 2-octanone & Moldy \\
\hline 10 & Methyl acetate & Green nuts \\
\hline 11 & Ethyl acetate & Slight bitter \\
\hline 12 & Ethyl propanoate & Strawberry, apple \\
\hline 13 & Hexyl acetate & Sweet \\
\hline 14 & (Z)-3-hexenyl acetate & Banana \\
\hline 15 & Methyl decanoate & Green leafs \\
\hline 16 & Hexanal & Cut grass, green \\
\hline 17 & (Z)-3-hexenal & Green leafs, apple \\
\hline 18 & (E)-2-pentenal & Grass, flowers \\
\hline 19 & (E)-2-hexenal & Almond \\
\hline 20 & $(2 \mathrm{E}, 4 \mathrm{E})$-hexa 2,4-dienal & Cut grass \\
\hline 21 & Hexen-1-ol & Fruity, aromatic \\
\hline 22 & (E)-3-hexen-1-ol & Green leaf, nut \\
\hline 23 & (Z)-3-hexen-1-ol & Banana \\
\hline 24 & (E)-2-hexen-1-ol & Green \\
\hline 25 & (Z)-2-hexen-1-ol & Fresh, green leafy \\
\hline 26 & 3-methylbutanol & Undesirable \\
\hline 27 & 2-methylbutanol & Undesirable \\
\hline 28 & 1-pentanol & Ripe fruits \\
\hline 29 & (Z)-2-pentenol & Green banana \\
\hline 30 & Benzyl alcohol & Violet flowers \\
\hline 31 & Ethanol & Ripe apple \\
\hline 32 & Acetic acid & Defect \\
\hline 33 & Propanoic acid & Pungent \\
\hline 34 & Isobutylic acid & Sweet \\
\hline 35 & Butanoic acid & Rancid \\
\hline 36 & Hexanoic acid & Mint \\
\hline 37 & (E)-2-hexenoic acid & Grass and fruity \\
\hline
\end{tabular}

fact that commercial oil is only one. With other commercial sampling, the classification zone of quality oils in the map could be compressed.

The structure of MLP-BP has 46 neurons in input, 110 neurons in the hidden layer and 112 output neurons. The supervised network utilizes the GC of each oil as input. The input data are encoded by the amplitude of 37 signals, whose intensities represent the components concentrations in the oil. The output neurons are 159, considering the parameters that request an evaluation. The number of the neurons in the hidden layer must be determined experimentally, because it is strictly connected to the case in study: in this case, the neurons in the hidden layer are 210. Few neurons can make the network unable to generate an adequate model, instead too much neutrons could create the "overfitting" event, which is an inadequate predisposition to generalisation. The output of the network must produce a series of findings about some of the flavours encoded by the PT. A correct training of the network is fundamental to create a model which was able to link input and output parameters. This neural network is structurally able to reproduce, with good accuracy, the classification of a panel starting from its GC. The learning error is about 0.008 .

It is well known that the ability of classification of ANN depends on the type of data and its structure, in the literature, there are very high values above the $95 \%$.

Then, if the GC result of a new oil sample is subjected to MLP-BP network, this will generate an output that will be, with good approximation, the one that would be generated by PT.

\section{Conclusions}

The regulation EEC 2568/91 and its upload [4] defined the procedures for the determination of the organoleptic quality of EVOO by introducing the tool of the PT. So, the assayers, as human beings, are custodians of the capacity to quantise the organoleptic quality in compliance with the ISO 9001 standard.

Considering the large increasing of EVOO consumption in Mediterranean countries and recently all over the world because of its delicious taste and aroma, the necessity to evaluate them is pressing. This suggested us to consider the PT as the depositary of a metrology "silent" system for the organoleptic quality, in fact, it works only when it is stimulated by samples of EVOO. 
Sweet

\begin{tabular}{|l|l|l|l|l|l|l|l|l|l|}
\hline \multicolumn{2}{|c|}{ F } & & & & & & c & \\
\hline f & & & & & A & & & & C \\
\hline & & & & a & & & & & \\
\hline e & & & & & & & & & \\
\hline E & & & & & & & & b & B \\
\hline & & & & & & & & & \\
\hline & & & & d & D & & & & \\
\hline & & & & & & & & & \\
\hline$\underline{i}$ & & & & & & & h & & \\
\hline & & I & & g & G & & & & H \\
\hline
\end{tabular}

Ripe

Fig. 5 Results of Kohonen map.

Spicy

Uppercase letters: trainer samples.

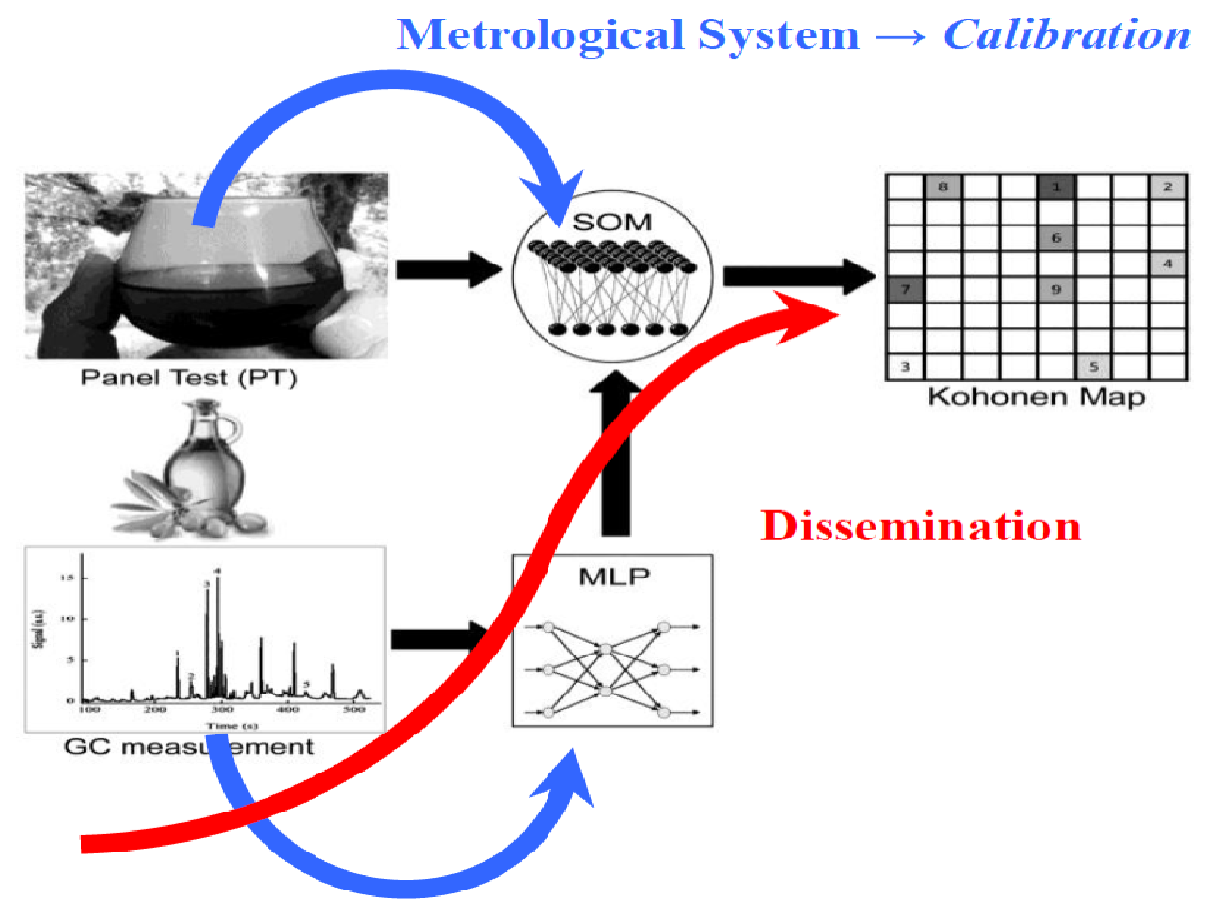

Fig. 6 Structure of metrologic dissemination system. 
The data of the PT have been used to train the Kohonen map with the purpose to make it more easily readable and highlight autonomously the clearest characteristics.

A later study will look for all the stimulation that could be mathematically "independent", allowing the eventual identification in PT of a "stimuli base system" that could be an equivalent of a measurement unit system of the flavour.

It is clear that the space of arbitrary output of the map of Kohonen is occupied as a function of the stimulation of the PT, but in text it has been observed that the "empirical priority" is respected in both of the classifications. These properties of Kohonen map have not been analysed and they will be the subject of a future work.

Now, this evaluation is realised by very expensive systems. A PT, performed according to the EU procedures, is hard because it requires accredited assayers. It is also an expensive process and, therefore, it is not easily accessible to the companies that have limited productions and if they were of high quality they might not have the correct market price.

From a technical point of view, the ANN architecture is robust to the possible variability of peaks of GC of each sample, because it is more sensible to the "signature" of GC, despite the variability of peaks intensity.

The identification of GC peaks does not seem to exclude the possibility that small signals could be instead determinants in sensory perception. This aspect can be revealed only through a wide and diversified stimulation of PT.

Knowing the capabilities of generalisation of MLP-BP, GC of a sample excluded from learning will likely to give an output which applied to the map of Kohonen, will place the sample in the map in a similar position to that in which it would have positioned the PT without that this has actually analysed.

This is the exact procedure of metrology dissemination extracted from PT. Then, a PT on a certain number of samples is undoubtedly the expression of an organoleptic metrology, incomplete but always improvable.

Finally, the developed tool is shown in Fig. 6, the cascade of the two ANNs and the MLP-BP are able to classify a sample different from those used for the learning of both the Kohonen map and the MLP-BP.

This system of classification has the advantage of a greater "readability" by consumers, than the PT results. They can directly read the original results. Common producers who can't easily reach a professional PT are aiming to provide access to it.

\section{Acknowledgments}

The realisation of this work has been possible thanks to some Italian EVOO producers. The author also wishes to thank "Flos Olei", the guide to the world best EVOO describing, popularising and promoting the ones that will excel in this selection.

\section{References}

[1] Quality Systems, Model for Quality Assurance in Design, Development, Production, Installation and Servicing, ISO 9001, 1994.

[2] R.M. Duran, Relationship between the composition and ripening of the olive and the quality of the oil, Acta Horticulturae 286 (1990) 441-451.

[3] C.M. Kalua, M.S. Allen, D.R. Bedgood, A.G. Bishop, P.D. Prenzler, K. Robards, Olive oil volatile compounds, flavour development and quality: A critical review, Food Chem. 100 (1) (2007) 273-286.

[4] Commission Regulation (EEC) No. 2568/91, http://www.politicheagricole.it/flex/cm/pages/ServeBLO B.php/L/IT/IDPagina/2884 (accessed Mar. 20, 2014).

[5] R. Garcia-Villalba, T. Pacchiarotta, A. Carrasco-Pancorbo, A. Segura-Carretero, A. Fernández-Gutiérrez, A.M. Deelder, et al., Gas chromatography - atmospheric pressure chemical ionization-time of flight mass spectrometry for profiling of phenolic compounds in extra virgin olive oil, Journal of Chromatography A 1218 (7) (2011) 959-971.

[6] M.C. Whitehead, C.S. Beeman, B.A. Kinsella, Distribution of taste and general sensory nerve endings in fungiform papillae of the hamster, American Journal of Anatomy 173 (3) (1985) 185-201.

[7] M.T. Morales, M. Tsimidou, The role of volatile 
compounds and polyphenols in olive oil sensory quality, in: J. Harwood, R. Aparicio (Eds.), Handbook of Olive Oil, Analysis and Properties, Aspen Publication, Gaithersburg, Maryland, 2000, pp. 393-458.

[8] International Olive Council, Testing methods, http://www.internationaloliveoil.org (accessed Mar. 20, 2014).

[9] B. Russell, The Principles of Mathematics, Cambridge University Press, Cambridge, 1903.

[10] F. Angerosa, Influence of volatile compounds on virgin olive oil quality evaluated by analytical approaches and sensor panels, European Journal of Lipid Science and Technology 104 (9-10) (2002) 639-660.

[11] D. Tura, P.D. Prenzler, D.R. Bedgood, J.M. Antolovich, K. Robards, Varietal and processing effects on the volatile profile of Australian olive oils, Food Chemistry 84 (3) (2004) 341-349.

[12] G. Luna, M.T. Morales, R. Aparicio, Characterization of 39 varietal virgin olive oils by their volatile composition, Food Chemistry 98 (2) (2006) 243-252.

[13] C. Pizarro, S. Rodríguez-Tecedor, N. Pérez-del-Notario, J.M. González-Sáiz, Recognition of volatile compounds as markers in geographical discrimination of Spanish extra virgin olive oils by chemometric analysis of non-specific chromatography volatile profiles, Journal of Chromatography A 1218 (3) (2011) 518-523.

[14] K. Gurney, An Introduction to Neural Networks, Routledge, London, 1997.
[15] S. Haykin, Neural Networks: A Comprehensive Foundation, Prentice Hall, Upper Saddle River, New Jersey, 1999.

[16] M. Oreggia, Flos Olei-A Guide to the World of Extra Virgin Olive Oil, E.V.O. srl, Rome, 2014. (in Italian)

[17] N.H. Snow, G.C. Slack, Head-space analysis in modern gas chromatography, TrAC Trends in Analytical Chemistry 21 (9-10) (2002) 608-617.

[18] M.T. Morales, M.V. Alonso, J.J. Rios, R. Aparicio, Virgin olive oil aroma: Relationship between volatile compounds and sensory attributes by chemometrics, Journal of Agriculture and Food Chemistry 43 (11) (1995) 2925-2931.

[19] S. Vichi, A.I. Castellote, L. Pizzale, L.S. Conte, S. Buxaderas, E. López-Tamames, Analysis of virgin olive oil volatile compounds by headspace solid-phase microextraction coupled to gas chromatography with mass spectrometric and flame ionization detection, Journal of Chromatography A 983 (1-2) (2003) 19-33.

[20] Neuro AI, Kohonen Self Organizing Maps, http://www.learnartificialneuralnetworks.com/kohonen.html (accessed Mar. 20, 2014).

[21] M. Caciotta, S. Giarnetti, F. Leccese, Hybrid neural network system for electric load forecasting of telecommunication station, in: Proceeding of XIX IMEKO World Congress-Fundamental and Applied Metrology, Lisbon, 2009.

[22] Agilent 7820A Gas Chromatograph Data Sheet, http://www.agilent.com (accessed Mar. 20, 2014). 